\title{
The use of different dialysis membranes in therapy of patients with multiple myeloma
}

\author{
Maciej Szymczak ${ }^{1, A-F}$, Dorota Zielińska ${ }^{2, D, F}$, Aleksandra Musiała ${ }^{1, D-F}$ \\ ${ }^{1}$ Department of Nephrology and Transplantation Medicine, Wroclaw Medical University, Poland \\ ${ }^{2}$ Department of Nephrology and Transplantation Medicine, University Clinical Hospital, Wrocław, Poland \\ A - research concept and design; $\mathrm{B}$ - collection and/or assembly of data; $\mathrm{C}$ - data analysis and interpretation; \\ $D$ - writing the article; $E$ - critical revision of the article; $F$ - final approval of the article
}

Address for correspondence

Maciej Szymczak

E-mail: maciej.szymczak@umed.wroc.pl

Funding sources

None declared

Conflict of interest

None declared

Received on February 5, 2020

Reviewed on April 25, 2020

Accepted on May 4, 2020

\begin{abstract}
Free light chains accumulation is the reason of kidney injury in patients with multiple myeloma. The removal of free light chains can improve patients prognosis and survival, and in some cases allows for dialysotherapy discontinuation. Unfortunately, conventional dialysis is not effective enough in terms of free light chains removal. New high cut-off ( $\mathrm{HCO}$ ) techniques remove free light chains more effectively than conventional dialysis. In some cases, this technique may turn out better than hemodiafiltration. However, there are some differences between specific techniques in the removal of kappa and lambda light chains. Lambda light chains are better removed by polymethyl methacrylate membranes with a change of filter during dialysis. Kappa light chains are thoroughly removed by polymethyl methacrylate membranes and HCO (35,000 Da) polysulfone membranes. Unfortunately, it is very difficult to differentiate between the effect of HCO dialysis therapy and concomitant chemotherapy because some of the data is not fully conclusive. Using the proper technique for an individual patient may give optimally effective treatment results.
\end{abstract}

Key words: treatment, multiple myeloma, dialysis membranes
Cite as

Szym czak M, Zielińska D, Musiała A. The use of different dialysis membranes in therapy of patients with multiple myeloma. Polim Med. 2019;49(2):67-70. doi:10.17219/pim/122014

DOI

10.17219/pim/122014

Copyright

(c) 2020 by Wroclaw Medical University

This is an article distributed under the terms of the

Creative Commons Attribution 3.0 Unported (CC BY 3.0)

(https://creativecommons.org/licenses/by/3.0/) 


\section{Introduction}

Conventional hemodialysis is unable to remove effectively the circulating free light chains in patients with multiple myeloma (MM). New high cut-off ( $\mathrm{HCO}$ ) dialysis techniques make it possible to decrease levels/concentrations of free light chains, especially with simultaneous chemotherapy treatment. Hemodiafiltration with ultrafiltrate regeneration on resin using 'super-high-flux' (polyphenylene super-high-flux (S-HF), with a nominal cut-off of $42 \mathrm{kD}$ ) membrane also effectively decreases free light chains concentrations. This kind of hemodiafiltration does not have any influence on albumin concentration. ${ }^{1}$ Using HCO membranes allows for stopping dialysis in some patients with MM (3 out of 5 in this study). ${ }^{2}$

Utilizing HCO membranes was associated with a higher rate of hemodialysis independence at 6 months $(56.5 \%$ HCO hemodialysis vs $35.4 \%$ conventional hemodialysis) and 12 months (60.9\% HCO hemodialysis vs $37.5 \%$ conventional hemodialysis). The frequency of adverse effects was similar in both groups (43\% of complications connected with hemodialysis in the HCO group in comparison to $39 \%$ in the conventional group), and mortality was similar in both groups. ${ }^{3}$

Similar results were achieved in terms of dialysis independency after 6 and 9 months of treatment when $\mathrm{HCO}$ dialysis membranes with bortezomib were used -6 out of 10 patients no longer needed dialysis, compared to 2 out of 10 patients undergoing conventional dialysis with bortezomib. ${ }^{4}$

Other authors found that in patients with MM, using $\mathrm{HCO}$ membranes results in lower mortality in comparison to conventional membranes. The survival rate after 1 year of treatment was $25 \%$ in the group of patients dialyzed using $\mathrm{HCO}$ dialyzer compared to $0 \%$ when a conventional dialysis was performed. Complete renal response rate, defined as an increase from $<50 \mathrm{~mL} / \mathrm{min}$ to $>60 \mathrm{~mL} / \mathrm{min}$ for at least 2 months, was $10.5 \%$ among patients treated with $\mathrm{HCO}$ dialyzers compared to $0 \%$ among patients treated with conventional dialysis. Partial renal response rate, defined as an increase of estimated glomerular filtration rate (eGFR) from $<15 \mathrm{~mL} / \mathrm{min}$ to $30-59 \mathrm{~mL} / \mathrm{min}$, was $15.8 \%$ and $5.3 \% \%$ in $\mathrm{HCO}$ dialysis- and conventional dialysis-treated patients, respectively, while minor renal response rate, defined as an increase from $<15 \mathrm{~mL} / \mathrm{min}$ to $15-29 \mathrm{~mL} / \mathrm{min}$ or from $15-29 \mathrm{~mL} / \mathrm{min}$ to $30-59 \mathrm{~mL} / \mathrm{min}$, was $26.3 \%$ and $15.8 \%$ in $\mathrm{HCO}$ dialysisand conventional dialysis-treated patients, respectively. Increased survival rate of patients and renal response rate correlated with a decrease of free light chains concentration. Total treatment costs were comparable in both groups of patients treated with usage of $\mathrm{HCO}$ membranes and conventional dialysis. ${ }^{5}$ While $\mathrm{HCO}$ membranes are more expensive than conventional ones, better overall treatment results lead to decreased total treatment costs, offsetting the higher price of $\mathrm{HCO}$ membranes. ${ }^{6}$

\section{Polymethylmethacrylate membranes}

The use of adsorptive membranes, such as polymethylmethacrylate-based BK-2.1 membrane, was also associated with better outcomes among patients with myeloma and cast nephropathy. ${ }^{7}$

Combining Theralite 2100 SUPRA device (Bellco, Mirandola, Italy), bortezomib and dexamethasone treatment resulted in a decrease of free light chains concentrations ranging from $72.8 \%$ to $99.7 \%$ in 3 weeks. Response to treatment was achieved in $80 \%$ of patients with acute kidney injury in the course of MM. ${ }^{8}$

High cut-off membranes dialysis effectively diminished free light chains concentrations in patients with acute kidney injury. A total of 11.6 six-hour HCO dialysis sessions per patient were performed, with free light chains decreasing by $93.7 \%$ in the course of treatment. Single dialysis session decreased free light chains by about $57.7 \% .^{9}$

On the other hand, a comparison of dialysis using polymethyl methacrylate membranes (PMMA), one of the most common HCO dialysis membranes, with conventional dialysis for patients simultaneously treated with bortezomib indicate no differences between these modalities after 3 months of treatment. The results of treatment were dependent on the hematologic response for treatment (56\% of patients with hematological response and $6.7 \%$ of patients without hematologic response were independent from dialysis). ${ }^{10}$

\section{Double polymethacrylate membranes}

Nonetheless, it was proved that the double polymethylmethacrylate filter (DELETE system) (Toray BK-F; Toray Industries, Inc., Tokyo, Japan) was effective in terms of free light chain removal in chronic dialysis patients with MM. ${ }^{11}$

Exchange of PMMA filter after $2 \mathrm{~h}$ of dialysis increased lambda light chains removal rates compared to classic PMMA, as it mitigated rapid PMMA saturation with free lambda chains, which diminishes their efficacy in removing the lambda chains. This kind of dialysis is called enhanced adsorption dialysis (EAD). The reason of this phenomenon is fast PMMA saturation with free lambda chains. Saturation of PMMA with free light chains decreases free lambda light chains removal efficacy.

This phenomenon was not observed in kappa light chains removal. It is suggested that the EAD method may be important in the treatment of MM patients with high concentrations of lambda light chains. ${ }^{12}$ High cut-off PMMA dialysis removes free light chains mainly through adsorption. In the course of hemodiafiltration, more free light chains are removed than in the used dialysis solution. High cut-off PMMA removes kappa light chains more effectively than hemodiafiltration. ${ }^{13}$ 


\section{Other membranes}

More effective clearance of kappa light chains compared to lambda light chains was observed also in $24 \mathrm{~h}$ dialysis using a HCO $(35,000 \mathrm{Da})$ polysulfone membrane. ${ }^{14}$ Light chains appear not only in MM; they are classified as free medium urea toxins. Comparison of free dialysis membranes: PMMA, polyphenylene HFR17 filter and conventional polysulfone filter F7HPS in terms of kappa and lambda free light chains removal indicates that PMMA and polyphenylene HFR17 filter are more effective than conventional polysulfone filter F7HPS. ${ }^{15}$

\section{Efficacy control}

It should be taken into account that dialysis membranes with nominally the same parameters may have a different efficacy of clearance. ${ }^{16}$ The structure of monoclonal protein is different in every patient and the real efficacy of free light chains should be controlled. Free light chains concentration should be checked every week of dialysis treatment. ${ }^{15}$ Actually, published data indicates that better prognosis is correlated with the extent of free light chain reduction in serum. ${ }^{5}$

\section{Controversies}

Contrary to the data indicating the superiority of $\mathrm{HCO}$ hemodialysis over conventional dialyzers, there are 2 multicenter randomized controlled trials in which this superiority was not confirmed. In MYRE study (98 participants and 48 hospitals in France), no statistically significant difference was shown in the hemodialysis independence rate between patients treated with $\mathrm{HCO}$ compared to conventional hemodialysis for 3 months ( $41.3 \%$ and $33 \%$, respectively, $\mathrm{p}=0.42$ ), although a significantly higher clearance of light chains in HCO dialyzers group was noticed. ${ }^{3}$ Significant difference in hemodialysis independence appeared 6 and 12 months of treatment $(56.5 \%$ vs $35.4 \%$ and $60.9 \%$ vs $37.5 \%$, respectively) but, as the authors of MYRE study concluded, these results should be considered exploratory. In EuLITE study (90 patients and 16 hospitals in UK and Germany), hemodialysis independence was observed in $56 \%$ of patients treated with $\mathrm{HCO}$ dialyzers and in $51 \%$ patients in the standard hemodialysis group $(p=0.81)$. More infections were observed as adverse events in $\mathrm{HCO}$ hemodialysis group, including lung infections (26 vs 13 infections, 14 vs 3 lung infections). ${ }^{17}$

\section{Summary}

The efficacy of HCO dialysis membranes in terms of diminishing free light chains resulted in guidelines of the International Myeloma Working Group Recommendations for the Diagnosis and Management of Myeloma-Related Renal Impairment: The use of HCO dialyzers in combination with anti-myeloma therapy should be considered (grade B). ${ }^{18}$ The use of $\mathrm{HCO}$ dialyzers in combination with anti-myeloma therapy seems to be a good option for treating patients with MM. However, future studies should precise the indications for this therapy.

\section{ORCID iDs}

Maciej Szymczak (D) https://orcid.org/0000-0002-1248-081X

Dorota Zielińska (D) https://orcid.org/0000-0003-1861-2622

Aleksandra Musiała (D) https://orcid.org/0000-0001-7358-2046

\section{References}

1. Menè $P$, Giammariolia $E$, Fofia $C$, et al. Serum free light chains removal by HFR hemodiafiltration in patients with multiple myeloma and acute kidney injury: A case series. Kidney Blood Press Res. 2018;43(4):1263-1272.

2. Peters NO, Laurain E, Cridlig J, Hulin C, Cao-Huu T, Frimat L. Impact of free light chain hemodialysis in myeloma cast nephropathy: A case-control study. Hemodial Int. 2011;15(4):538-545.

3. Bridoux F, Carron PL, Pegourie B, et al; MYRE Study Group. Effect of high-cutoff hemodialysis vs conventional hemodialysis on hemodialysis independence among patients with myeloma cast nephropathy: A randomized clinical trial. JAMA. 2017;318(21):2099-2110.

4. Buus NH, Rantanen JM, Krag SP, Andersen NF, Jensen JD. Hemodialysis using high cut off filters in light chain cast nephropathy. Blood Purif. 2015;40(3):223-231.

5. Curti A, Schwarz A, Trachsler J, Tomonaga Y, Ambühl PM. Therapeutic efficacy and cost effectiveness of high cut-off dialyzers compared to conventional dialysis in patients with cast nephropathy. PLoS One. 2016;11(7):e0159942. doi:10.1371/journal.pone.0159942

6. Florens N, Juillard L. Expanded haemodialysis: News from the field. Nephrol Dial Transplant. 2018;33(Suppl 3):iii48-iii52.

7. Sens $F$, Chaintreuil $D$, Jolivot $A$, et al. Effectiveness of IHD with adsorptive PMMA membrane in myeloma cast nephropathy: A cohort study. Am J Nephrol. 2017;46(5):355-363.

8. Buti E, Dervishi E, Ghiandai G, et al. Free light chains reduction on acute kidney injury in multiple myeloma: Critical role of high cutoff membranes [In Italian]. G Ital Nefrol. 2014;31(6):gin/31.6.11.

9. Berni Wennekers A, Martín Azara MP, Dourdil Sahun V, et al. Thirteen treated of acute renal failure secondary to multiple myeloma with high cut off filters. Nefrologia. 2016;36(4):418-426.

10. Hudier L, Decaux O, Haddj-Elmrabet A, et al. Intensive haemodialysis using PMMA dialyser does not increase renal response rate in multiple myeloma patients with acute kidney injury. Clin Kidney $J$. 2018;11(2):230-235.

11. Santoro A, Grazia M, Mancini E. The double polymethylmethacrylate filter (DELETE system) in the removal of light chains in chronic dialysis patients with multiple myeloma. Blood Purif. 2013;35(Suppl 2):5-13.

12. Fabbrini P, Sirtori S, Casiraghi E, et al. Polymethylmethacrylate membrane and serum free light chain removal: Enhancing adsorption properties. Blood Purif. 2013;35(Suppl 2):52-58.

13. Oshihara W, Nagao H, Megano H, Arai J, Koide M, Takada M. Trial use of a polymethylmethacrylate membrane for the removal of free immunoglobulin light chains in dialysis patients. NDT Plus. 2010;3(Suppl 1):i3-i7.

14. Hanf W, Guillaume C, Jolivot A, et al. Prolonged hemodialysis for acute kidney injury in myeloma patients. Clin Nephrol. 2010;74(4):319-322.

15. Donati G, Moretti MI, Baraldi O, et al. Removal of free light chains in hemodialysis patients without multiple myeloma: A crossover comparison of three different dialyzers. BMC Nephrol. 2016;17(1):193.

16. Ouseph R, Hutchison CA, Ward RA. Differences in solute removal by two high-flux membranes of nominally similar synthetic polymers. Nephrol Dial Transplant. 2008;23(5):1704-1712. 
17. Hutchison CA, Cockwell P, Moroz V, et al. High cutoff versus highflux haemodialysis for myeloma cast nephropathy in patients receiving bortezomib-based chemotherapy (EuLITE): A phase 2 randomised controlled trial. Lancet Haematol. 2019;6(4):e217-e228.

18. Dimopoulos MA, Sonneveld P, Leung N, et al. International Myeloma Working Group Recommendations for the Diagnosis and Management of Myeloma-Related Renal Impairment. J Clin Oncol. 2016;34(13):1544-1557. 